\title{
On $H^{\infty}$ Well-Posed Cauchy Problems for Some Weakly Hyperbolic Pseudo-differential Equations
}

\author{
by \\ Yasutoshi S HIOZAKI ${ }^{*}$
}

\section{§1. Introduction}

We start with the initial value problems for a class of nonlinear equations which W. Craig [1] mentioned. He treated the following problem

$$
\begin{gathered}
\partial_{t}^{2} u+F\left(\partial_{1} H u, \partial_{t} u, u ; x, t\right)=0, \quad t \in[0, T], \quad x \in \mathbb{R}^{1}, \\
u\left(t_{0}, x\right)=u_{0}(x), \quad \partial_{t} u\left(t_{0}, x\right)=u_{1}(x), \quad x \in \mathbb{R}^{1},
\end{gathered}
$$

where the initial time $t_{0}$ is a constant in $[0, T]$, and the operator $H$ is the Hilbert transform, that is,

$$
H u(x)=\frac{1}{\pi} \mathrm{v} \cdot \mathrm{p} \cdot \int \frac{u(y)}{x-y} d y
$$

Note that $H=-i \operatorname{sgn}(D)$, where $\operatorname{sgn}(D)$ is a Fourier multiplier operator which is

$$
\begin{gathered}
\operatorname{sgn}(D) u(x)=\frac{1}{2 \pi} \iint \operatorname{sgn}(\xi) u(y) e^{(1-1) \xi} d y d \xi, \\
\operatorname{sgn}(\xi)=\left\{\begin{aligned}
1, & \xi>0 \\
-1, & \xi<0,
\end{aligned}\right.
\end{gathered}
$$

so we have

$$
\partial_{1} H u(x)=|D| u(x)=\frac{1}{2 \pi} \iint|\xi| u(y) e^{(\imath-1) \xi} d y d \xi .
$$

He says that the above equation is non-strictly hyperbolic since the linearized equation

Communicated by S. Matsuura, May 20, 1992.

1991 Mathematics Subject Classifications: 35L15, 35L80, 35S10.

* Department of Applied Mathematics and Phisics, Faculty of Engineering, Kyoto University, Kyoto 606, Japan. 


$$
\partial_{t}^{2} v+\frac{\partial F}{\partial\left(\partial_{x} H u\right)} \partial_{x} H v+\frac{\partial F}{\partial\left(\partial_{t} u\right)} \partial_{t} v+\frac{\partial F}{\partial u} v=g
$$

has double characteristics (Observe that only the first term of the left-hand side is second order and the rest is smaller order.) and showed that the initial value problem is well-posed (locally in time) for Sobolev initial data under the condition that

$$
\frac{\partial F}{\partial\left(\partial_{x} H u\right)}(s) \geq \delta>0 \quad(\delta: \text { constant })
$$

for all values of $s=\left(\partial_{x} H u, \partial_{t} u, u ; x, t\right)$ under consideration. (This type equation appears in a theory of fluid dynamics, for example, H. Yosihara [11]. See also T. Nishida [9].)

In order to see the essence of Craig's argument, we consider here the following linear equation

$$
\left(\partial_{t}^{2}+a(t, x)|D|+b(t, x) \partial_{t}+c(t, x)\right) u(t, x)=f(t, x),
$$

where $a, b$ and $c$ are $C^{\infty}$ functions whose derivatives up to arbitrary order are bounded in $[0, T] \times \mathbf{R}^{1}$. (Recall that if we replace the term $a(t, x)|D|$ in the above equation by $a(t, x) \partial_{x}$, then the equation would be not $H^{\infty}$ well-posed unless $a(t, x) \equiv 0$, by Levi's condition. See in detail S. Mizohata and Y. Ohya [8].)

If we assume

$$
a(t, x) \geq \delta>0, \quad \delta: \text { constant }, \quad t \in[0, T], \quad x \in \mathbf{R}^{1},
$$

(This assumption corresponds to (1.8).) then we can lead an energy-inequality for the following energy-norm

$$
\operatorname{Re}(a(t, x)|D| u(t, x), u(t, x))_{s}+C\|u(t, x)\|_{s}^{2}+\left\|\partial_{t} u(t, x)\right\|_{s}^{2},
$$

where ( , ), and \|\| , are $H^{\prime}$-inner product and norm with respect to $x \in \mathbf{R}^{1}$ respectively, and that this energy-norm is equivalent to

$$
\left\||D|^{\frac{1}{2}} u(t, x)\right\|_{,}^{2}+C\|u(t, x)\|_{s}^{2}+\left\|\partial_{t} u(t, x)\right\|_{1}^{2} .
$$

These results give $H^{\infty}$ well-posedness for (1.9) and (1.2). The form of (1.12) suggests that (1.9) should be $H^{\infty}$ well-posed even if the term which includes $|D|^{\frac{1}{2}} u$ is added to the left-hand side, and it is not difficult to verify this.

Moreover Y. Hattori and Y. Ohya [2] treated an example in which merely $a(t, x) \geq 0$ is satisfied instead of (1.10). The case they analyzed is that the initial time is fixed on $t_{0}=0$ and that the equation has the following form

$$
\partial_{t}^{2} u+t^{2 k}|D| u+\alpha t^{\prime}|D|^{\frac{1}{2}} u=0, \quad t \in[0, T], \quad x \in \mathbf{R}^{1},
$$




$$
u(0, x)=u_{0}(x), \quad \partial_{t} u(0, x)=u_{1}(x), \quad x \in \mathbf{R}^{1},
$$

where $k, l$ are non-negative integers and $\alpha$ is a real constant. They derived that $H^{\infty}$ well-posedness of (1.13) and (1.14) is equivalent to the condition that either $\alpha<0$ and $l+1 \geq k$ or $\alpha \geq 0$ and $k, l$ are arbitrary. (This result is similar to the one for the following differential equation

$$
\left(\partial_{t}^{2}-t^{2 k} \partial_{x}^{2}+\alpha t^{l} \partial_{x}\right) u(t, x)=0, \quad t \in[0, T], \quad x \in \mathbf{R}^{1} .
$$

The necessary and sufficient condition of $H^{\infty}$ (or $C^{\infty}$ ) well-posedness for (1.15) and (1.14) is that either $\alpha \neq 0$ and $l+1 \geq k$ or $\alpha=0$ and $k, l$ are arbitrary. See in detail Mizohata [5] p.13, Theorem 4.)

In this paper we consider at first the following initial value problem

$$
\begin{gathered}
\left(\partial_{t}^{2}+a(t, x)|D|^{m}+b(t, x)|D|^{n}\right) u(t, x)=f(t, x), \quad t \in[0, T], \quad x \in \mathbf{R}^{d}, \\
u\left(t_{0}, x\right)=u_{0}(x), \quad \partial_{t} u\left(t_{0}, x\right)=u_{1}(x), \quad x \in \mathbf{R}^{d},
\end{gathered}
$$

with arbitrary initial time $t_{0} \in[0, T]$. Here $m, n$ are real constants such that $2 \geq m>n>0$ and $a(t, x), b(t, x)$ are as before except for being complex valued. (Our argument shall be independent of the number of the space variables. And every term that includes $\partial_{t} u$ or $u$ is omitted since it is not essential.)

Under the assumption (1.10) we can study (1.16) using the energy-norm

$$
\operatorname{Re}\left(a(t, x)|D|^{m} u(t, x), u(t, x)\right)_{s}+C\|u(t, x)\|_{s}^{2}+\left\|\partial_{t} u(t, x)\right\|_{s}^{2},
$$

or some variations. It can be seen that the above norm is equivalent to

$$
\left\||D|^{\frac{m}{2}} u(t, x)\right\|_{s}^{2}+C\|u(t, x)\|_{s}^{2}+\left\|\partial_{t} u(t, x)\right\|_{,}^{2},
$$

and that (1.16) is $H^{\infty}$ well-posed in the case $m / 2 \geq n$. Moreover even if $n>m / 2$ and $b(t, x)$ is real-valued, then we can find such a variation of the energy-norm (1.18) that follows $H^{\infty}$ well-posedness. (See Theorem 2.2.) On the other hand, we would expect that the Cauchy problem is not $H^{\infty}$ well-posed when $n>m / 2$ and $b(t, x)$ is non-real. At the end of $\S 2$ we will consider the constant-coefficient case, where it is not difficult to prove the above assertion. This type restriction never appears in the case of hyperbolic differential operators.

Moreover we analyze the following equation

$$
\left(\partial_{t}^{2}+t^{2 k}|D|^{m}+\alpha t^{\prime}|D|^{n}\right) u(t, x)=0 \quad t \in[0, T], \quad x \in \mathbf{R}^{d}
$$

with the initial data at $t_{0}=0$. (Here $k, l$ are as before and $\alpha$ is a complex constant.) We shall give the necessary and sufficient condition of $H^{\infty}$ wellposedness for the above equation. (See Theorem 3.2.)

Finally the author would like to thank Professors Y. Ohya and S. Tarama for their many interesting suggestions on these problems. 


\section{§2. Hil Well-posedness of the Weakly Hyperbolic Equation (1.16)}

As mentioned in the preceding section, we consider here $H^{\infty}$ well-posedness of the Cauchy problem (1.16) and (1.17). In the equations the initial time $t_{0}$ is a constant in $[0, T], a(t, x), b(t, x)$ are in $C^{\infty}\left([0, T] ; \mathscr{B}^{\infty}\left(\mathbb{R}^{d}\right)\right)\left(\mathscr{B}^{\infty}\left(\mathbb{R}^{d}\right)\right.$ denotes the set of $C^{\infty}$ functions whose derivatives up to arbitrary order are bounded in $\mathbb{R}^{d}$.) and the operator $|D|^{m}$ with respect to $x$ is defined by

$$
\left.\left|D^{m} u(x) \equiv \frac{1}{(2 \pi)^{d}} \iint\right| \xi\right|^{m} u(y) e^{\imath(x-y) \xi} d y d \xi
$$

for $m>0$ and $u(x) \in C_{0}^{\infty}\left(\mathbb{R}^{d}\right)$. Note that $|D|^{m}$ can be extended to a continuous linear mapping from $H^{\text {s+m }}\left(\mathbb{R}^{d}\right)$ to $H^{s}\left(\mathbb{R}^{d}\right)$ for every $s \in \mathbb{R}$.

Moreover we assume

$$
2 \geq m>n>0 .
$$

Before analyzing the equation (1.16) we must define the precise meaning of $H^{\infty}$ well-posedness.

Definition 2.1. The Cauchy problem for (1.16) is called uniformly $H^{\infty}$ well posed when if any $t_{0} \in[0, T], u_{0}(x), u_{1}(x) \in H^{\infty}\left(\mathbb{R}^{d}\right)$ and $f(t, x) \in C^{\infty}([0, T])$; $\left.H^{\infty}\left(\mathbb{R}^{d}\right)\right)$ be given, then there is a unique solution $\left.u(t, x) \in C^{\infty}([0, T]) ; H^{\infty}\left(\mathbb{R}^{d}\right)\right)$ of (1.16) and (1.17).

In this section we consider uniformly $H^{\infty}$ well-posedness of (1.16) in the case where $a(t, x) \geq \delta>0$.

Theorem 2.2. Let $a(t, x), b(t, x) \in C^{\infty}\left([0, T] ; \mathscr{B}^{\infty}\left(\mathbb{R}^{d}\right)\right)$ be a real-valued and $a$ complex-valued function, respectively. Assume (2.2) and that there is $\delta>0$ such that

$$
a(t, x) \geq \delta>0, \quad \text { for any }(t, x) \in[0, T] \times \mathbb{R}^{d} .
$$

Then (i) if $m / 2 \geq n$ then the Cauchy problem (1.16) is uniformly $H^{\infty}$ well-posed. (ii) if $n>m / 2$ and $b(t, x)$ is real-valued, then the Cauchy problem (1.16) is uniformly $H^{\infty}$ well-posed.

The proof of Theorem 2.2 is not difficult but needs much description, so we only sketch it here.

Case (i): For any $s \in \mathbb{R}$ we define the following norm and energy.

$$
\begin{gathered}
E_{1}(t) \equiv\|u(t, x)\|_{\frac{m}{2}+s}^{2}+\left\|\partial_{t} u(t, x)\right\|_{?}^{2}, \\
E_{1,}(t) \equiv \operatorname{Re}\left(a(t, x)|D|^{m} u(t, x), u(t, x)\right),+C\|u(t, x)\|_{3}^{2}+\left\|\partial_{t} u(t, x)\right\|_{,}^{2},
\end{gathered}
$$


where $C$ is an appropriate positive constant, and $(,)_{s}$ and \|\|$_{s}$ denote $H^{s}$ inner product and norm with respect to $x \in \mathbb{R}^{d}$, respectively. By (2.2) and (2.3) it is obvious that there are $C_{1}, C_{2}>0$ such that

$$
C_{1} \cdot E_{s}(t) \leq E_{1, s}(t) \leq C_{2} \cdot E_{s}(t)
$$

for any $t \in[0, T]$ and $u(t, x) \in C^{1}\left([0, T] ; H^{s+\frac{m}{2}}\right)$, using the ordinary Garding inequality. ( $|D|^{m}$ is not exactly a pseudo-differential operator since its symbol $|D|^{m}$ has a singularity on $\xi=0$, but we can treat $|D|^{m}$ similarly as a pseudodifferential operator after modifying its symbol in a neighborhood of the origin. This modification gives no serious influence to our argument.)

The following inequality will be derived from $(1.16),(2.2),(2.5),(2.6)$, and that $m / 2 \geq n$.

$$
\begin{aligned}
\left|\frac{d}{d t} E_{1, s}(t)\right| & \leq \text { const } \cdot\left(E_{1, s}(t)+\|f(t, \cdot)\|_{s}^{2}\right) \\
& \text { for any } \left.t \in[0, T] \text { and } u(t, x) \in C^{1}([0, T]) ; H^{1+\frac{m}{2}}\right) .
\end{aligned}
$$

In particular, the assumption (2.2) is important in order to estimate the commutators of some operators, and the assumption that $m / 2 \geq n$ is used so as to estimate the term $b(t, x)|D|^{n}$.

From (2.6) and (2.7) we get the following energy-inequality

$$
E_{\mathrm{s}}(t) \leq \mathrm{const} \cdot\left(E_{\mathrm{s}}(0)+\int_{0}^{t}\|f(\tau, \cdot)\|^{2}, d \tau\right) \quad \text { for every } t \in[0, T]
$$

and this proves the assertion (i) by applying Riesz's representation theorem on the Sobolev spaces.

Case (ii): We must modify the energy-norm as follows.

$$
\begin{aligned}
E_{2, s}(t) \equiv & \operatorname{Re}\left(\left\{a(t, x)|D|^{m}+b(t, x)|D|^{n}\right\} u(t, x), u(t, x)\right), \\
& +C\|u(t, x)\|_{\mathrm{s}}^{2}+\left\|\partial_{t} u(t, x)\right\|^{2} .
\end{aligned}
$$

We can lead the inequality which be gotten by replacing $E_{1, s}(t)$ by $E_{2.1}(t)$ in (2.6), from (2.2) and (2.3). And an energy-inequality corresponding to (2.8) also follows since we have the assumption that $b(t, x)$ is real. The rest is the same as Case (i).

Thus Theorem 2.2 follows.

We can prove the same result even in adding the terms which include $\partial_{t} u(t, x)$ or $u(t, x)$ to the left-hand side of $(1.16)$. Such terms request no serious modification to the proof of $H^{\infty}$ well-posedness. (Hence we may regard the above result as an extension of the one for (1.9).) On the other hand, the term $b(t, x)|D|^{n}$ can be treated as a 'harmless lower order term' only if $m / 2 \geq n$ or $b(t, x)$ is real.

If $n>m / 2$ and $b(t, x)$ is not real, then is not the Cauchy problem for (1.16) $H^{\infty}$ well-posed? Here we consider the constant-coefficient case, where the 
Fourier image of the equation is important. The full symbol of the operator in the left-hand side of (1.16) is

$$
-\tau^{2}+a|\xi|^{m}+b|\xi|^{n}
$$

and the zeros with respect to $\tau$ can be written as follows.

$$
\begin{aligned}
\pm \sqrt{a|\xi|^{m}+b|\xi|^{n}} & = \pm \sqrt{a}|\xi|^{\frac{m}{2}}\left(1+\frac{b}{a}|\xi|^{n-m}\right)^{\frac{1}{2}} \\
& \approx \pm \sqrt{a}|\xi|^{\frac{m}{2}}\left(1+\frac{b}{2 a}|\xi|^{n-m}\right) .
\end{aligned}
$$

So the imaginary part of the zeros are near $\pm(\operatorname{Im} b /(2 \sqrt{a}))|\xi|^{n-\frac{m}{2}}$ for large $|\xi|$, and they grow as a positive power of $|\xi|$ under the assumption that $a>0, \operatorname{Im} b \neq 0$ and $n-m / 2>0$. Therefore we can show that the Cauchy problem is not $H^{\infty}$ wellposed under this condition by the same method as that for usual constantcoefficient differential equations. (c.f. S. Mizohata [6], Theorem 4.6 and 5.2.)

\section{§3. $H^{\infty}$ Well-posedness of the Weakly Hyperbolic Equation (1.20)}

In this section we consider the Cauchy problem for (1.16) under the assumption that $a(t, x) \geq 0$ for any $(t, x) \in[0, T] \times \mathbf{R}^{d}$, instead of (2.3).

Here we are interested in the case where $a(t, x), b(t, x)$ degenerate in a finite order at some point $\left(t_{0}, x_{0}\right)$. One of the most essential examples in this situation is the following Cauchy problem mentioned at the end of $\S 1$.

$$
\begin{gathered}
\left(\partial_{t}^{2}+t^{2 k}|D|^{m}+\alpha t^{l}|D|^{n}\right) u(t, x)=0, \quad t \in[0, T], \quad x \in \mathbb{R}^{d}, \\
u(0, x)=u_{0}(x), \quad \partial_{t} u(0, x)=u_{1}(x), \quad x \in \mathbb{R}^{d} .
\end{gathered}
$$

Here $k, l$ are non-negative integers, $m, n$ are real constants such that $m>n>0$, and $\alpha$ is a complex constant. This equation is an extension of (1.13). For the simplicity we consider only homogeneous case (i.e. $f(t, x) \equiv 0)$ and restrict the initial time to $t_{0}=0$. So here we define $H^{\infty}$ well-posedness as follows.

Definition 3.1. The Cauchy problem for (3.1) is called $H^{\infty}$ well-posed when if every $u_{0}(x), u_{1}(x) \in H^{\infty}\left(\mathbf{R}^{d}\right)$ be given, then there is a unique solution $u(t, x)$ $\in C^{\infty}\left([0, T] ; H^{\infty}\left(\mathbf{R}^{d}\right)\right)$ of (3.1) and (3.2).

As we suggested in $\S 1$, the situation when $m / 2 \geq n$ is different from that when $n>m / 2$. The necessary and sufficient condition of $H^{\infty}$ well-posedness for (3.1) is as follows.

Theorem 3.2. Under the above assumption, the following is valid.

I. If $\alpha \geq 0$, then the Cauchy problem (3.1) is always $H^{\infty}$ well-posed. 
II. If $\alpha<0$, then $H^{\infty}$ well-posedness of (3.1) is equivalent to that $\frac{m}{2 n} \geq \frac{k+1}{l+2}$.

III. If $\operatorname{Im} \alpha \neq 0$ (i.e. $\alpha$ is non-real) and $m / 2 \geq n$, then $H^{\infty}$ well-posedness of (3.1) is equivalent to that $\frac{m}{2 n} \geq \frac{k+1}{l+2}$.

IV. If $\operatorname{Im} \alpha \neq 0$ and $m / 2<n$, then the Cauchy problem for (3.1) is not $H^{\infty}$ well-posed.

The most interesting difference from (1.13) or the differential equation (1.15) is the result in the case IV, which corresponds to the fact that we mentioned at the end of the preceding section.

The remainder of this paper is devoted to the proof of Theorem 3.2. We consider the Fourier image of (3.1) with respect to $x$, that is,

$$
\left(\partial_{t}^{2}+t^{2 k}|\xi|^{m}+\alpha t^{l}|\xi|^{n}\right) v(t, \xi)=0 \quad \text { on } t \in[0, T] \text { and } \xi \in \mathbf{R}^{d}
$$

The above is an ordinary differential equation with respect to $t$ with a parameter $\xi$, so the solution $v(t, \xi)$ exists for every initial data $v(0, \xi)$ and $\partial_{t} v(0, \xi)$. Now the following lemma is valid.

Lemma 3.3. $H^{\infty}$ well-posedness for (3.1) is equivalent to that there exist positive constants $C$ and $p$ such that the following inequality is satisfied; for every solution $v(t, \xi)$ of $(3.3)$,

$$
E_{0}(t, \xi) \leq C|\xi|^{p} E_{0}(0, \xi) \quad \text { for any } t \in[0, T] \text { and large }|\xi| \text {. }
$$

Here we define

$$
E_{0}(t, \xi) \equiv|v(t, \xi)|^{2}+\left|\partial_{t} v(t, \xi)\right|^{2}
$$

The above lemma is due to I. G. Petrowsky.

Lemma 3.4. Let $C_{0}$ be an arbitrary positive constant. Then

$$
E_{0}\left(t^{\prime}, \xi\right) \leq \text { const } \cdot E_{0}(t, \xi) \quad \text { for any } t, t^{\prime} \in\left[0, t_{0}(\xi)\right] \text { and large }|\xi|,
$$

where

$$
t_{0}(\xi) \equiv C_{0}|\xi|^{-m}
$$

and the constant in (3.6) is independent of $t, \xi$ and $v(\cdot, \cdot)$.

Proof. We rewrite (3.3) as follows. 


$$
\frac{\partial}{\partial t}\left[\begin{array}{c}
v(t, \xi) \\
\partial_{t} v(t, \xi)
\end{array}\right]=\left[\begin{array}{cc}
0 & 1 \\
-t^{2 k \mid}|\xi|^{m}-\alpha t^{\prime}|\xi|^{n} & 0
\end{array}\right]\left[\begin{array}{c}
v(t, \xi) \\
\partial_{t} v(t, \xi)
\end{array}\right] .
$$

All elements of the matrix in the right-hand side is bounded by const $\cdot|\xi|^{m}$. Thus

$$
\left|\partial_{t} E_{0}(t, \xi)\right| \leq \text { const } \cdot|\xi|^{m} E_{0}(t, \xi) .
$$

This implies (3.6).

Lemma 3.5. Assume that $2 k>l$. Let $C_{1}, C_{2}$ be arbitrary constants and define

$$
\left\{\begin{array}{l}
t_{1}(\xi) \equiv C_{1}|\xi|^{-\sigma_{1}}, \quad \sigma_{1} \equiv \frac{m-n}{2 k-l}(>0), \\
t_{2}(\xi) \equiv C_{2}|\xi|^{-\sigma_{2}}, \quad \sigma_{2} \equiv \frac{n / 2}{l / 2+1}(>0) .
\end{array}\right.
$$

Then the following estimate is valid for the solution $v(t, \xi)$ of (3.3).

$$
E_{0}(t, \xi) \leq \text { const } \cdot|\xi|^{p_{0}} E_{0}(0, \xi),
$$

$$
E_{0}(t, \xi) \leq \text { const } \cdot|\xi|^{p_{0}} E_{0}\left(\min \left(t_{1}(\xi), t_{2}(\xi)\right), \xi\right),
$$

both for any $t \in\left[0, \min \left(t_{1}(\xi), t_{2}(\xi)\right)\right]$ and large $|\xi|$, where $p_{0}$ is some positive constant independent of $t, \xi$ and $v(\cdot, \cdot)$.

Proof. Rewrite (3.3) as follows.

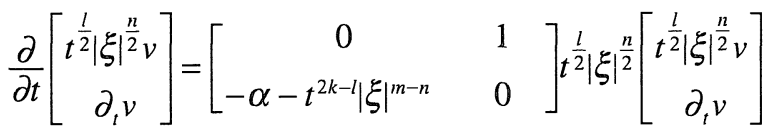

$$
\begin{aligned}
& +\frac{l}{2 t}\left[\begin{array}{ll}
1 & 0 \\
0 & 0
\end{array}\right]\left[\begin{array}{c}
t^{\frac{1}{2}}|\xi|^{\frac{n}{2}} v \\
\partial_{t} v
\end{array}\right] \text { for } t \in(0, T]
\end{aligned}
$$

Now that $2 k>l$ and (3.9) imply that

$$
t^{2 k-1}|\xi|^{m-n} \leq t_{1}(\xi)^{2 k-l}|\xi|^{m-n}=C_{1}^{2 k-1} \quad \text { when } t \leq t_{1}(\xi) .
$$

This and (3.12) lead the following inequality,

$$
\left|\partial_{t} E_{1}(t, \xi)\right| \leq \mathrm{const} \cdot\left(t^{\frac{l}{2}}|\xi|^{\frac{n}{2}}+t^{-1}\right) E_{1}(t, \xi) \quad \text { for } t \in\left(0, t_{1}(\xi)\right],
$$

where

$$
\left.\left.E_{1}(t, \xi) \equiv\left|t^{\frac{1}{2}}\right| \xi\right|^{\frac{n}{2}} v(t, \xi)\right|^{2}+\left|\partial_{t} v(t, \xi)\right|^{2} .
$$

And 


$$
t^{\frac{l}{2}}|\xi|^{\frac{n}{2}}+t^{-1} \leq t^{-1}\left(t_{2}(\xi)^{\frac{l}{2}+1}|\xi|^{\frac{n}{2}}+1\right) \leq \mathrm{const} \cdot t^{-1} \quad \text { when } t \leq t_{2}(\xi),
$$

so

$$
\left|\partial_{t} E_{1}(t, \xi)\right| \leq \frac{\gamma}{t} \cdot E_{1}(t, \xi) \quad \text { for } t \in\left(0, \min \left(t_{1}(\xi), t_{2}(\xi)\right)\right]
$$

where $\gamma$ is a suitable positive constant. This follows

$$
E_{1}(t, \xi) \leq\left(\frac{t}{t^{\prime}}\right)^{\gamma} E_{1}\left(t^{\prime}, \xi\right) \quad \text { for } t, t^{\prime} \in\left(0, \min \left(t_{1}(\xi), t_{2}(\xi)\right)\right] \quad \text { with } t^{\prime} \leq t,
$$

and this leads

$$
E_{1}(t, \xi) \leq \mathrm{const} \cdot|\xi|^{\sigma \gamma} E_{1}\left(|\xi|^{-\sigma}, \xi\right) \text { for } t \in\left[|\xi|^{-\sigma}, \min \left(t_{1}(\xi), t_{2}(\xi)\right)\right],
$$

where $\sigma$ is a positive constant larger than $\sigma_{1}, \sigma_{2}$ and $m$. Noting that

$$
\begin{gathered}
E_{1}(t, \xi) \leq \text { const } \cdot|\xi|^{p_{1}} E_{0}(t, \xi), \quad E_{0}(t, \xi) \leq \text { const } \cdot|\xi|^{p_{2}} E_{1}(t, \xi) \\
\text { for any } t \in\left[|\xi|^{-\sigma}, T\right] \text { and large }|\xi|,
\end{gathered}
$$

we obtain

$$
E_{0}(t, \xi) \leq \mathrm{const} \cdot|\xi|^{p_{0}} E_{0}\left(|\xi|^{-\sigma}, \xi\right) \quad \text { for } t \in\left[|\xi|^{-\sigma}, \min \left(t_{1}(\xi), t_{2}(\xi)\right)\right] \text { and large }|\xi|,
$$

and this proves (3.10) with Lemma 3.4. (3.11) is also derived by the same argument.

\section{\$4. The Proof of Theorem 3.2: the Case I, II and III}

In the following argument we divide the results of Theorem 3.2 into several lemmas and prove them all.

Lemma 4.1. Assume that either $\alpha<0$ or $\alpha$ is non-real. Moreover, if

$$
\frac{m}{2 n}<\frac{k+1}{l+2}
$$

is satisfied, then the Cauchy problem for (3.1) is not $H^{\infty}$ well-posed.

Proof. Note that $1 \leq m / n$ and $m / n<(2 k+2) /(l+2)$ imply $2 k>l$. Let us consider (3.12). We can take $t^{2 k-l}|\xi|^{m-n}$ as small as we please when $t \leq t_{1}(\xi)$, taking $C_{1}$ sufficiently small. Then the most important part of (3.12) is the matrix $\left[\begin{array}{cc}0 & 1 \\ -\alpha & 0\end{array}\right]$. The eigenvalues of this matrix are $\pm \sqrt{-\alpha}$, the real parts of which are non-zero when either $\alpha<0$ or $\alpha$ is non-real. We choose such a branch of the square roots that $\operatorname{Re} \sqrt{-\alpha}>0$. 
Multiplying (3.12) by a costant non-singular matrix which diagonalizes the above matrix, we have the following form

(4.1) $\frac{\partial}{\partial t}\left[\begin{array}{l}W_{0}(t, \xi) \\ W_{1}(t, \xi)\end{array}\right]=\left[\begin{array}{cc}\sqrt{-\alpha}+\varepsilon_{11} & \varepsilon_{12} \\ \varepsilon_{21} & -\sqrt{-\alpha}+\varepsilon_{22}\end{array}\right] t^{\frac{l}{2}}|\xi|^{\frac{n}{2}}\left[\begin{array}{l}W_{0}(t, \xi) \\ W_{1}(t, \xi)\end{array}\right]+t^{-1} P_{0}\left[\begin{array}{l}W_{0}(t, \xi) \\ W_{1}(t, \xi)\end{array}\right]$,

where $P_{0}$ is some constant matrix and $\varepsilon_{i j}(t, \xi)(i, j=1,2)$ is as small as we please when $t \leq t_{1}(\xi)$, by choosing $C_{1}$. Defining

$$
E_{W}(t, \xi) \equiv\left|W_{0}(t, \xi)\right|^{2}+\left|W_{1}(t, \xi)\right|^{2}, \quad S_{W}(t, \xi) \equiv\left|W_{0}(t, \xi)\right|^{2}-\left|W_{1}(t, \xi)\right|^{2},
$$

we obtain for some positive constant $\delta_{1}$,

$$
\begin{aligned}
\partial_{t} S_{W}(t, \xi) \geq & \delta_{1} t^{\frac{l}{2}}|\xi|^{\frac{n}{2}} E_{W}(t, \xi)-\text { const } \cdot t^{-1} E_{W}(t, \xi) \\
& \text { for any } t \in\left(0, t_{1}(\xi)\right] \text { and large }|\xi| .
\end{aligned}
$$

Note that the assumption $m / 2 n<(k+1) /(l+2)$ implies $\sigma_{1}<\sigma_{2}$ and $t_{1}(\xi)>t_{2}(\xi)$ for large $|\xi|$. Taking $C_{2}$ sufficiently large, (4.3) leads

$$
\partial_{t} S_{W}(t, \xi) \geq \delta_{2} t^{\frac{l}{2}}|\xi|^{\frac{n}{2}} E_{W}(t, \xi) \geq \delta_{2} t^{\frac{l}{2}}|\xi|^{\frac{n}{2}} S_{W}(t, \xi) \quad \text { for any } t \in\left[t_{2},(\xi), t_{1}(\xi)\right] .
$$

By integrating this, we have for some positive $\delta_{t}(i=1,2)$ and $\rho$,

$$
\begin{aligned}
S_{W}\left(t_{1}(\xi), \xi\right) & \geq \exp \left[\delta_{2}|\xi|^{\frac{n}{2}} \int_{t_{2}(\xi)}^{t_{1}(\xi)} t^{\frac{l}{2}} d t\right] S_{W}\left(t_{2}(\xi), \xi\right) \\
& \geq \exp \left[\delta_{3}|\xi|^{\rho}\right] \cdot S_{W}\left(t_{2}(\xi), \xi\right) .
\end{aligned}
$$

Now we shall show that (4.4) contradicts $H^{\infty}$ well-posedness. Solve (3.3) giving the following initial data,

$$
W_{0}\left(t_{2}(\xi), \xi\right)=1, \quad W_{1}\left(t_{2}(\xi), \xi\right)=0
$$

at $t=t_{2}(\xi)$. Then $E_{W}\left(t_{2}(\xi), \xi\right)=S_{W}\left(t_{2}(\xi), \xi\right)=1$. Suppose that the Cauchy problem is $H^{\infty}$ well-posed. Then (3.4) is valid, so

$$
E_{W}\left(t_{1}(\xi), \xi\right) \leq \mathrm{const} \cdot|\xi|^{p_{1}} E_{0}\left(t_{1}(\xi), \xi\right) \leq \mathrm{const} \cdot|\xi|^{p+p_{1}} E_{0}(0, \xi) .
$$

On the other hand, Lemma 3.5 says

$$
E_{0}(0, \xi) \leq \text { const } \cdot|\xi|^{p_{0}} E_{0}\left(t_{2}(\xi), \xi\right) \leq \text { const } \cdot|\xi|^{p_{0}+p_{2}} E_{W}\left(t_{2}(\xi), \xi\right) .
$$

Taking $|\xi| \rightarrow \infty,(4.4)-(4.7)$ imply a contradiction.

Lemma 4.2. If $m / 2 \geq n$ and $m / 2 n \geq(k+1) /(l+2)$, then the Cauchy problem (3.1) is $H^{\infty}$ well-posed.

Proof. Rewrite (3.3) as follows. 


$$
\frac{\partial}{\partial t}\left[\begin{array}{c}
|\xi|^{\frac{m}{2}} v \\
\partial_{t} v
\end{array}\right]=\left[\begin{array}{cc}
0 & 1 \\
-t^{2 k} & 0
\end{array}\right]|\xi|^{\frac{m}{2}}\left[\begin{array}{c}
|\xi|^{\frac{m}{2}} v \\
\partial_{t} v
\end{array}\right]+\left[\begin{array}{cc}
0 & 0 \\
-\alpha t^{l}|\xi|^{n-\frac{m}{2}} & 0
\end{array}\right]\left[\begin{array}{c}
|\xi|^{\frac{m}{2}} v \\
\partial_{t} v
\end{array}\right]
$$

Letting

$$
N(t) \equiv\left[\begin{array}{rr}
-i t^{k}-1 \\
-i t^{k} & 1
\end{array}\right], \quad U(t, \xi)=\left[\begin{array}{c}
U_{0}(t, \xi) \\
U_{1}(t, \xi)
\end{array}\right] \equiv N(t)\left[\begin{array}{r}
|\xi|^{\frac{m}{2}} v \\
\partial_{t} v
\end{array}\right],
$$

it follows from (4.8) that

$$
\begin{aligned}
\partial_{t} U(t, \xi)= & i t^{k}\left[\begin{array}{rr}
1 & 0 \\
0 & -1
\end{array}\right]|\xi|^{\frac{m}{2}} U(t, \xi)+\frac{k}{2 t}\left[\begin{array}{ll}
1 & 1 \\
1 & 1
\end{array}\right] U(t, \xi) \\
& -\frac{i}{2} \alpha t^{l-k}|\xi|^{n-\frac{m}{2}}\left[\begin{array}{rr}
-1 & -1 \\
1 & 1
\end{array}\right] U(t, \xi) \quad \text { for any } t \in(0, T]
\end{aligned}
$$

Now $t^{l-k}|\xi|^{n-\frac{m}{2}} \leq t^{l+1-k} \cdot t^{-1}$ for large $|\xi|$ because $n-m / 2 \leq 0$. Thus it is bounded by const $\cdot t^{-1}$ if $l+1-k \geq 0$. In this case we obtain

$$
\left|\partial_{t} E_{U}(t, \xi)\right| \leq \text { const } \cdot t^{-1} E_{U}(t, \xi) \quad \text { for any } t \in(0, T] \text { and large }|\xi|,
$$

where $E_{U}(t, \xi)=\left|U_{0}(t, \xi)\right|^{2}+\left|U_{1}(t, \xi)\right|^{2}$. (4.10) derives (3.4) by the same argument that treated $E_{1}(t, \xi)$ in the proof of Lemma 3.5.

Next we consider the case where $l+1-k<0$, which implies $m / 2>n$. (If $m / 2=n$, then that $m / 2 n=1 \geq(k+1) /(l+2)$ leads $l+1-k \geq 0$.) Giving an arbitrary positive constant $C_{3}$, define

$$
t_{3}(\xi) \equiv C_{3}|\xi|^{\sigma_{3}}, \quad \sigma_{3} \equiv \frac{\frac{m}{2}-n}{k-l-1}(>0) .
$$

Then

$$
\begin{aligned}
t^{l-k}|\xi|^{n-\frac{m}{2}} \leq & t_{3}(\xi)^{-(k-l-1)}|\xi|^{n-\frac{m}{2}} \cdot t^{-1}=C_{3}^{-(k-l-1)} \cdot t^{-1} \\
& \text { for any } t \in\left[t_{3}(\xi), T\right] \text { and large }|\xi|,
\end{aligned}
$$

so we can find the energy-inequality on this interval by the previous argument. Finally we consider the interval $\left[0, t_{3}(\xi)\right]$. That $k-l-1>0$ implies that $2 k>l$, so $t_{1}(\xi)$ in (3.9) is well-defined. Moreover $m / 2 n \geq(k+1) /(l+2)$ implies $\sigma_{3} \geq \sigma_{1} \geq \sigma_{2}$, so $t_{3}(\xi) \leq t_{1}(\xi) \leq t_{2}(\xi)$ is satisfied. Thus the interval $\left[0, \min \left(t_{1}(\xi)\right.\right.$, $\left.\left.t_{2}(\xi)\right)\right]=\left[0, t_{1}(\xi)\right]$ includes $\left[0, t_{3}(\xi)\right]$. Therefore (3.4) follows from Lemma 3.5.

Lemma 4.3. If $\alpha$ is real and $m / 2 n \geq(k+1) /(l+2)$, then the Cauchy problem for (3.1) is $H^{\infty}$ well-posed.

Proof. Defining 


$$
\Lambda(t, \xi) \equiv \sqrt{t^{2 k}|\xi|^{m}+\alpha t^{l}|\xi|^{n}}
$$

we rewrite (3.3) as follows.

$$
\frac{\partial}{\partial t}\left[\begin{array}{l}
\Lambda v \\
\partial_{t} v
\end{array}\right]=\left[\begin{array}{rr}
0 & 1 \\
-1 & 0
\end{array}\right] \Lambda\left[\begin{array}{l}
\Lambda v \\
\partial_{t} v
\end{array}\right]+\frac{\partial_{t} \Lambda}{\Lambda}\left[\begin{array}{ll}
1 & 0 \\
0 & 0
\end{array}\right]\left[\begin{array}{l}
\Lambda v \\
\partial_{t} v
\end{array}\right]
$$

Multiplying (4.13) by a constant non-singular matrix, we get the following form,

$$
\frac{\partial}{\partial t}\left[\begin{array}{l}
V_{0}(t, \xi) \\
V_{1}(t, \xi)
\end{array}\right]=i\left[\begin{array}{rr}
1 & 0 \\
0 & -1
\end{array}\right] \Lambda(t, \xi)\left[\begin{array}{l}
V_{0} \\
V_{1}
\end{array}\right]+\frac{\partial_{t} \Lambda}{\Lambda} \cdot P_{1} \cdot\left[\begin{array}{l}
V_{0} \\
V_{1}
\end{array}\right]
$$

where $P_{1}$ is a suitable constant matrix.

Now we let

$$
\tilde{t}_{1}(\xi) \equiv\left\{\begin{array}{l}
t_{1}(\xi)=C_{1}|\xi|^{-\sigma_{1}}, \text { if } 2 k>l \\
t_{0}(\xi)=C_{0}|\xi|^{-m}, \text { if } 2 k \leq l
\end{array}\right.
$$

and take $C_{1}$ sufficiently large if $2 k>l$. Then

$$
\frac{1}{2} t^{2 k}|\xi|^{m} \geq|\alpha| t^{l}|\xi|^{n} \quad \text { for any } t \in\left[\tilde{t}_{1}(\xi), T\right]
$$

which leads that $\Lambda(t, \xi)$ is real in this interval, and that

$$
\left|\frac{\partial_{t} \Lambda}{\Lambda}\right|=\left|\frac{2 k t^{2 k-1}|\xi|^{m}+l \alpha t^{l-1}|\xi|^{n}}{2\left(t^{2 k}|\xi|^{m}+\alpha t^{l}|\xi|^{n}\right)}\right| \leq \frac{t^{-1}(2 k+l) t^{2 k}|\xi|^{m}}{2 \cdot \frac{1}{2} t^{2 k}|\xi|^{m}} \leq \text { const } \cdot t^{-1}
$$

Therefore we can obtain the energy estimate in the interval $\left[\tilde{t}_{1}(\xi), T\right]$ by the same argument that treats $E_{1}$ in the proof of Lemma 3.5. On the estimate in $\left[0, \tilde{t}_{1}(\xi)\right]$, Lemma 3.4 can be applied when $2 k \leq l$ (i.e. $\tilde{t}_{1}(\xi)=t_{0}(\xi)$ ). If $2 k>l$, then recalling $m / 2 n \geq(k+1) /(l+2)$ implies $\tilde{t}_{1}(\xi)=t_{1}(\xi) \leq t_{2}(\xi)$, we can apply Lemma 3.5.

Lemma 4.4. If $\alpha \geq 0$, then the Cauchy problem for (3.1) is $H^{\infty}$ well-posed.

Proof. Under this assumption, $\Lambda(t, \xi)$ in (4.12) is always real. Moreover

$$
\left|\frac{\partial_{t} \Lambda}{\Lambda}\right|=\frac{2 k t^{2 k-1}|\xi|^{m}+l \alpha t^{l-1}|\xi|^{n}}{2\left(t^{2 k}|\xi|^{m}+\alpha t^{l}|\xi|^{n}\right)} \leq \frac{\text { const }}{t} \quad \text { for any } t \in(0, T]
$$

which immediately shows the estimate (3.4) by the previous argument. 


\section{§. The Proof of Theorem 3.2: the Final Case}

Finally we shall prove the result of the case IV. Note that we can omit the case $m / 2 n<(k+1) /(l+2)$ since Lemma 4.1 is already proved.

Lemma 5.1. Assume $1>m / 2 n \geq(k+1) /(l+2)$. Moreover if $\operatorname{Im} \alpha \neq 0$, then the Cauchy problem for (3.1) is not $H^{\infty}$ well-posed.

Proof. Step-1. We consider (4.14). Letting $\alpha=\alpha_{1}+i \alpha_{2}\left(\alpha_{1}\right.$ and $\alpha_{2}$ are real and $\alpha_{2} \neq 0$ ), we have

$$
\frac{\partial_{t} \Lambda}{\Lambda}=\frac{2 k t^{2 k-1}|\xi|^{m}+l \alpha_{1} t^{l-1}|\xi|^{n}+i l \alpha_{2} t^{l-1}|\xi|^{n}}{2\left(t^{2 k}|\xi|^{m}+\alpha_{1} t^{l}|\xi|^{n}+i \alpha_{2} t^{l}|\xi|^{n}\right)} .
$$

Then

$$
\left|\frac{i l \alpha_{2} t^{l-1}|\xi|^{n}}{t^{2 k}|\xi|^{m}+\alpha_{1} t^{l}|\xi|^{n}+i \alpha_{2} t^{l}|\xi|^{n}}\right|=\frac{l\left|\alpha_{2}\right| t^{l-1}|\xi|^{n}}{\left[\left(t^{2 k}|\xi|^{m}+\alpha_{1} t^{l}|\xi|^{n}\right)^{2}+\left(\alpha_{2} t^{l}|\xi|^{n}\right)^{2}\right]^{\frac{1}{2}}} \leq \frac{l}{t}
$$

and

$$
\begin{aligned}
\left|\frac{2 k t^{2 k-1}|\xi|^{m}+l \alpha_{1} t^{l-1}|\xi|^{n}}{t^{2 k}|\xi|^{m}+\alpha_{1} t^{l}|\xi|^{n}+i \alpha_{2} t^{l}|\xi|^{n}}\right| & =\frac{\left|2 k t^{-1}\left(t^{2 k}|\xi|^{m}+\alpha_{1} t^{l}|\xi|^{n}\right)\right|+\left.\left|(l-2 k) t^{-1} \alpha_{1} t^{l}\right| \xi\right|^{n} \mid}{\left[\left(t^{2 k}|\xi|^{m}+\alpha_{1} t^{l}|\xi|^{n}\right)^{2}+\left(\alpha_{2} t^{l}|\xi|^{n}\right)^{2}\right]^{\frac{1}{2}}} \\
& \leq \text { const } \cdot t^{-1},
\end{aligned}
$$

so $\left|\partial_{t} \Lambda / \Lambda\right|$ is bounded by const $\cdot t^{-1}$.

Step-2. Expanding the square root in (4.12), we get

$$
\Lambda(t, \xi)= \pm t^{k}|\xi|^{\frac{m}{2}}\left(1+\frac{\alpha}{2} \cdot t^{l-2 k}|\xi|^{n-m}+\lambda_{0}(\xi)\right)
$$

where the sign depends on the choice of the square root in (4.12). And

$$
\left|\lambda_{0}(\xi)\right| \leq \varepsilon t^{l-2 k}|\xi|^{n-m},
$$

where $\varepsilon$ becomes as small as we please when $t^{l-2 k}|\xi|^{n-m}$ is sufficiently small. Recalling the argument in the proof of Lemma 4.3 , we can choose such a branch of the square root in (4.12) that the following should be satisfied for some positive constant $\delta_{5}$.

$$
-\operatorname{Im} \Lambda(t, \xi) \geq \delta_{5} t^{l-k}|\xi|^{n-\frac{m}{2}} \text { for any } t \in\left[\tilde{t}_{1}(\xi), T\right] .
$$

Now letting

$$
E_{V}(t, \xi) \equiv\left|V_{0}(t, \xi)\right|+\left|V_{1}(t, \xi)\right|, \quad S_{V}(t, \xi) \equiv\left|V_{0}(t, \xi)\right|-\left|V_{1}(t, \xi)\right|,
$$


we can obtain

$$
\begin{aligned}
\partial_{t} S_{V}(t, \xi) \geq & \delta_{6} t^{l-k}|\xi|^{n-\frac{m}{2}} E_{V}(t, \xi)-\text { const } \cdot t^{-1} E_{V}(t, \xi) \\
& \text { for any } t \in\left[\tilde{t}_{1}(\xi), T\right] .
\end{aligned}
$$

Here we use the result of Step-1.

Step-3. Noting the assumption $1>m / 2 n \geq(k+1) /(l+2)$ implies $l+1-k$ $>0$, we can define $\sigma_{3}>0$ by (4.11) again. Thus we obtain from (5.5),

$$
\begin{aligned}
\partial_{t} S_{V}(t, \xi) \geq & \delta_{6} t^{l-k}|\xi|^{n-\frac{m}{2}} S_{V}(t, \xi) \\
& \text { for any } t \in\left[\max \left(t_{0}(\xi), t_{3}(\xi)\right), T\right] \text { and large }|\xi|
\end{aligned}
$$

taking $C_{3}$ sufficiently large if necessary. Here we use the fact that if $2 k>l$ then $m / 2 n \geq(k+1) /(l+2)$ implies $\sigma_{1} \geq \sigma_{3}$, i.e. $t_{1}(\xi) \leq t_{3}(\xi)$.

Defining $\tilde{t}(\xi) \equiv \max \left(t_{0}(\xi), t_{3}(\xi)\right)$, the integration of (5.6) gives us

$$
\begin{aligned}
S_{V}(T, \xi) & \geq \exp \left[\delta_{6}|\xi|^{n-\frac{m}{2}} \int_{\tilde{t}(\xi)}^{T} t^{l-k} d t\right] \cdot S_{V}(\tilde{t}(\xi), \xi) \\
& \geq \exp \left[\delta_{7} T^{l+1-k}|\xi|^{n-\frac{m}{2}}\right] \cdot S_{V}(\tilde{t}(\xi), \xi) .
\end{aligned}
$$

Now we shall show that the estimate (3.4) of Lemma 3.3 contradicts (5.7), giving a suitable initial data of $V_{0}$ and $V_{1}$ on $t=\tilde{t}(\xi)$. (Recall the proof of Lemma 4.1.) For that it is sufficient that the following estimate should be given.

$$
E_{0}(0, \xi) \leq \text { const } \cdot|\xi|^{p} E_{V}(\tilde{t}(\xi), \xi) \text { for large }|\xi| \text {. }
$$

If $\tilde{t}(\xi)=t_{0}(\xi)$, then the estimate (5.8) is reduced to Lemma 3.4. Therefore the rest is the case $\tilde{t}(\xi)=t_{3}(\xi)$.

Step-4. We assume that $\tilde{t}(\xi)=t_{3}(\xi)$. Here we may also assume $\tilde{t}_{1}(\xi)$ $\leq t_{3}(\xi)$. Return to (5.1) and get

$$
\begin{aligned}
|\operatorname{Im} \Lambda(t, \xi)| \leq & \text { const } \cdot t^{l-k}|\xi|^{n-\frac{m}{2}} \leq \text { const } \cdot t^{-1} \\
& \text { for any } t \in\left[\tilde{t}_{1}(\xi), t_{3}(\xi)\right] \text { and large }|\xi| .
\end{aligned}
$$

This leads the following estimate with (4.14).

$$
E_{V}\left(\tilde{t}_{1}(\xi), \xi\right) \leq \mathrm{const} \cdot|\xi|^{p_{7}} E_{V}\left(t_{3}(\xi), \xi\right) \text { for large }|\xi| \text {. }
$$

If $2 k \leq l$, then $\tilde{t}_{1}(\xi)=t_{0}(\xi)$ and the estimate (5.8) can be given by (5.9) and Lemma 3.4. In the case where $2 k>l$, we have $\tilde{t}_{1}(\xi)=t_{1}(\xi) \leq t_{2}(\xi)$ which shows (5.8) using (5.9) and Lemma 3.5.

Thus the proof of Theorem 3.2 is complete. 


\section{References}

[1] Craig, W., Nonstrictly hyperbolic nonlinear systems, Math. Ann., 277 (1987), 213-230.

[2] Hattori, Y. and Ohya, Y., On a differential operator appearing in the analysis of water waves, Math. Jap., 36 (1991), 591-601.

[3] Hörmander, L., The Cauchy problem for differential equations with double characteristics, $J$. Analyse Math., 32 (1977), 118-196.

[4] Mizohata, S., Some remarks on the Cauchy problem, J. Math. Kyoto Univ., 1 (1961), 109-127.

[5] - On the Cauchy problem, Science Press and Academic Press, Inc., 1985.

[6] - The theory of partial differential equations, Cambridge Univ. Press, 1973.

[7] Mizohata, S. and Ohya, Y., Sur la condition de E. E. Levi concernant des équations hyper boliques, Publ. RIMS, Kyoto Univ., 4 (1968/9), 511-526.

[8] - Sur la condition d'hyperbolicité pour les équations à caractéristiques multiples II, Japan. J. Math., 40 (1971), 63-104.

[9] Nishida, T., Analysis of equations of fluid (in Japanese), Sûgaku, 37 (1985), 289-304.

[10] Oleinik, O.A., On the Cauchy problem for weakly hyperbolic equations, Comm. Pure Appl. Math., 23 (1970), 569-586.

[11] Yosihara, H., Gravity waves on the free surface of an incompressible perfect fluid of finite depth, Publ. RIMS, Kyoto Univ., 18 (1982), 49-96. 
